\title{
Cluster Head Optimization based on Gravitational Search Algorithm
}

\author{
Ekta Singh \\ JMIT, Radaur
}

\author{
Vikas Gupta \\ JMIT, Radaur
}

\begin{abstract}
Wireless Sensor Networks (WSN) consist of nodes with limited power deployed in the area of interest. Nodes cooperate to collect, transmit and forward data to a base station. The major challenges of WSN are restricted energy supply, informatics addressing method is not possible and has lesser information measure and memory capability. In present work attempt has been made to reduce overall energy consumption of the network also reducing the bandwidth and memory requirements by using an optimization algorithm i.e. Gravitational Search Algorithm (GSA). Results will be compared with another optimization algorithm i.e. Genetic Algorithm.
\end{abstract}

\section{General Terms}

Security, Energy Consumption, Memory Capacity, Bandwidth

\section{Keywords}

Cluster Head, Genetic Algorithm, Gravitational Search Algorithm, Wireless Sensor Networks.

\section{INTRODUCTION}

Recently, technological advances in the design of processors, memory and radio communications have impelled an active interest in the area of distributed sensor networking, in which a number of independent, self-sustainable nodes collaborate to perform information gathering and processing in real time. Networks of such devices are commonly referred to as Wireless Sensor Networks (WSNs), which are envisioned as a bridge between the modern broadband packet data networks and the physical world. WSNs have made possible real-time data aggregation and analysis on an unprecedented scale [1]. Hence, they have attracted attention and garnered widespread appeal towards applications in diverse areas including disaster warning systems, environment monitoring, health care, safety and strategic areas such as defense reconnaissance, surveillance, and intruder detection. In case of nuclear power plant if any small delay occurs for data forwarding due to any node failure may results in severe disaster [2]. Hence effective Topology Control is required to obtain an energy efficient sensor network even if any node fails. An energy efficient topology control using gravity inspired algorithm based cluster head selection is presented in this work. A two tier architecture of WSN which consists of nodes and cluster heads is considered for simulation purpose. Residual Energy, Bandwidth and Memory Capacity are used as selection criteria for cluster head by proposed algorithm. The gravitational search algorithm (GSA) is used to select cluster head. Network is divided into number of clusters, which we have taken as $5 \%$ of the total number of nodes of a network [3]. Nodes are assigned to the cluster having minimum distance to the cluster head having maximum energy. The distance is calculated using Euclidean Distance Formula [4]

\section{PROPOSED WORK}

The present work is based upon the minimization of the energy consumption in WSN by selecting cluster head in two tier topology of WSN and optimum path selection from nodes to cluster head. For this purpose, the cluster head with maximum residual energy, bandwidth and memory capacity is selected amongst nodes after initial HELLO message transfer to every nodes within range [5]. For this purpose gravitational search algorithm (GSA) is used because the election of cluster head is a NP hard problem and also bounded by many constraints. Once the $\mathrm{CHs}$ were elected, data forwarding using minimum transmission power through its $\mathrm{CH}$ neighbors to the sink node is done [6]. Probability to become a $\mathrm{CH}$ is more for a node with maximum $\mathrm{RE}$, maximum unused $\mathrm{BW}$ and maximum unused memory. If any two of the parameters remain with maximum value and other one with minimum value, then probability of that node to become $\mathrm{CH}$ depends on the weights given for that parameter. Using Spearman's Rank Correlation Coefficient, the weights of the node parameters are identified. The parameters are namely distance, RSSI, residual energy, bandwidth and memory capacity which is inherited form [7].

Based on the relationship between the parameters, weights were assigned and a fitness function is formulated as follows,

$$
f(x)=0.2 \times 1+0.3 \times 2+0.5 \times 3
$$

The three parameters BW, Memory capacity and RE, considered for $\mathrm{CH}$ selection are represented by the variables $\mathrm{x} 1, \mathrm{x} 2$ and $\mathrm{x} 3$.

A step by step algorithm for the proposed work is given as:

STEP1. Initialize the random positions of agents in GSA

STEP2. Consider the searching space dimension as number of total binary digits for bandwidth, energy and memory which is equal to 25 .

STEP3. Calculate the fitness function for each agent

STEP4. Compare the fitness value of each agent with the previous best position of agent. If fitness function value is less for this new position than previous position then it will be assigned as new.

STEP5. The current position selected in previous step is used to get the mass for each agent as per GSA algorithm. The minimum value of fitness function is selected as best and maximum as worst position and using the formulas, mass of each agent can be calculated as: 


$$
\begin{gathered}
m_{i}(t)=\frac{f i t(t)-\operatorname{worst}(t)}{\operatorname{best}(t)-\operatorname{worst}(t)} \\
M_{i}(t)=\frac{m_{i}(t)}{\sum_{j=1}^{N} m_{j}(t)}
\end{gathered}
$$

STEP6. Gravitational force is calculated as:

$$
F_{i j}^{d}(t)=G(t) \cdot\left(M_{p i}(t) \times \frac{M_{a i}(t)}{R_{i j}(t)}+\varepsilon\right) \cdot\left(x_{j}^{d}(t)-x_{i}^{d}(t)\right)
$$

STEP7. This acceleration is calculated in GSA as

$$
a_{i}^{d}(t)=F_{i}^{d}(t) / M_{i i}(t) .
$$

STEP8. Add the new velocity to old position of particles and get the new updated positions which is conserved towards the minimization of objective function.

STEP9. Above all steps repeats till iterations last.

STEP10. Result will be positions of particles with minimum fitness function output. These positions are binary digits for remaining bandwidth, memory and energy for cluster heads [8].

Following these steps in optimization of GSA an optimal network for training is constructed in our work.

The root soul of this work is its objective function, on the basis of which optimal values of input weights and biases are set. We have developed a different MATLAB module which is called for each particle in each iteration. In the objective function module, the binary strings of each parameter is converted to decimal by using the equation

$$
x_{i} \frac{x^{u}-x^{l}}{2^{\beta}-1} \sum_{j=0}^{\beta} \gamma_{i} 2^{j} \quad ; i=1,2,3
$$

Where $x^{l}$ is the lower bound of the variable $x_{i}, x^{u}$ is the upper bound of the variable $x_{i}, \beta$ is the length of the string representing the variable and $\gamma \mathrm{j}$ represents the bit value of the jth position. For the obtained variables $\mathrm{x} 1$, $\mathrm{x} 2$ and $\mathrm{x} 3$, the fitness function is calculated for maximizing the function given in equation 1.1 .

\section{RESULTS}

Initialization of proposed optimization requires many constants to tune manually for better results.

These values are shown in table 1:

\section{Table 1. Initialization parameters of GSA}

\begin{tabular}{|c|c|}
\hline Number of Agents & 10 \\
\hline Number of iterations & 100 \\
\hline Initial Gravitational constant & 100 \\
\hline Alpha & 23 \\
\hline
\end{tabular}

For results analysis we have initialized 3 parameters of the nodes which are:

Table 2: Initialization values of WSN nodes parameters

\begin{tabular}{|c|c|c|}
\hline Bandwidth & Memory & Energy \\
\hline $250(\mathrm{kbps})$ & $128 \mathrm{~kb}$ & $1024 \mathrm{~J}$ \\
\hline
\end{tabular}

Keeping the other factors constant at some value, only number of nodes is changed.

Below are the optimization curves for GSA and GA by using MATLAB environment.

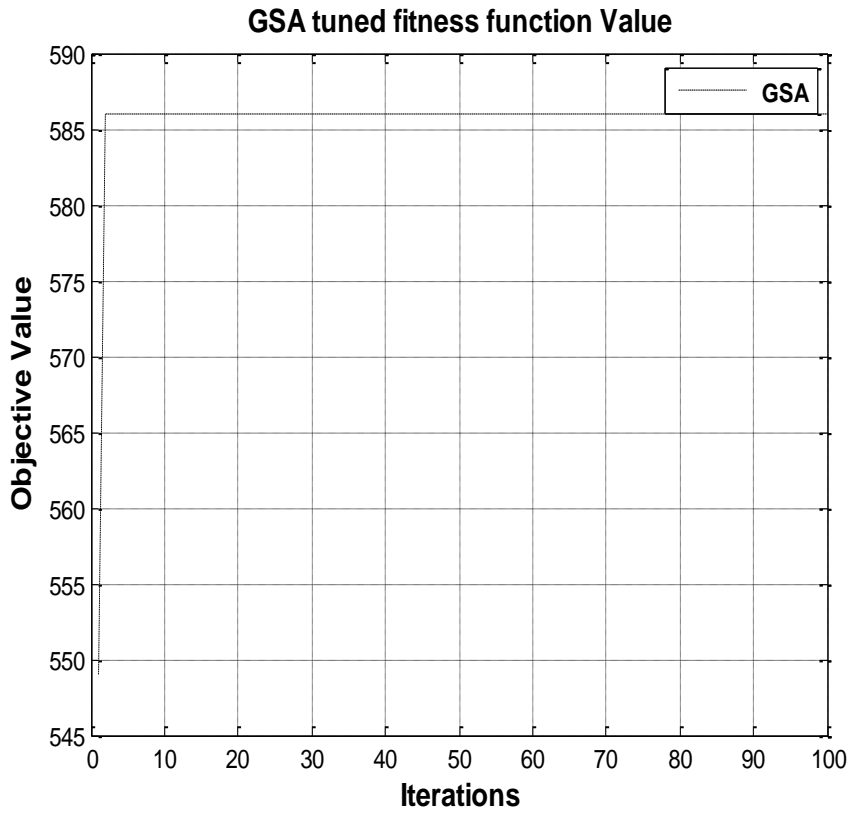

Fig. 1: Optimization curve for proposed algorithm

The earlier it settles to a maximum value as in this case, good is the optimization.

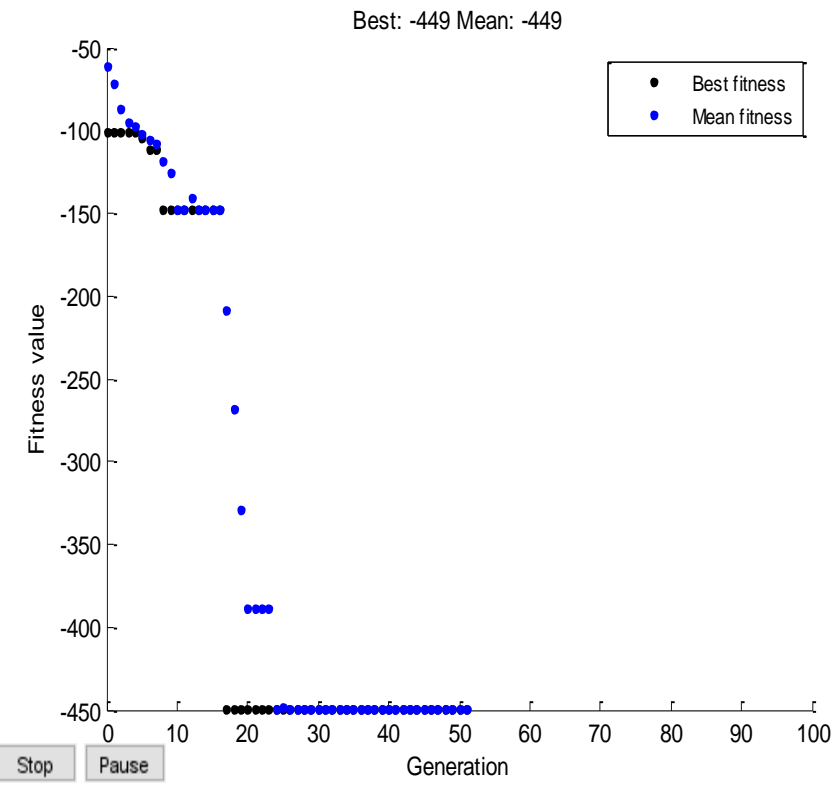

Fig. 2: Optimization curve for Genetic algorithm for cluster head selection

The objective function used is negative for Genetic Algorithm as the Genetic Algorithm toolbox of MATLAB works for minimization and for maximization, a negative function has to be used. 
Below are the comparison graphs of GA and GSA for Bandwidth, Memory and Residual energy respectively.

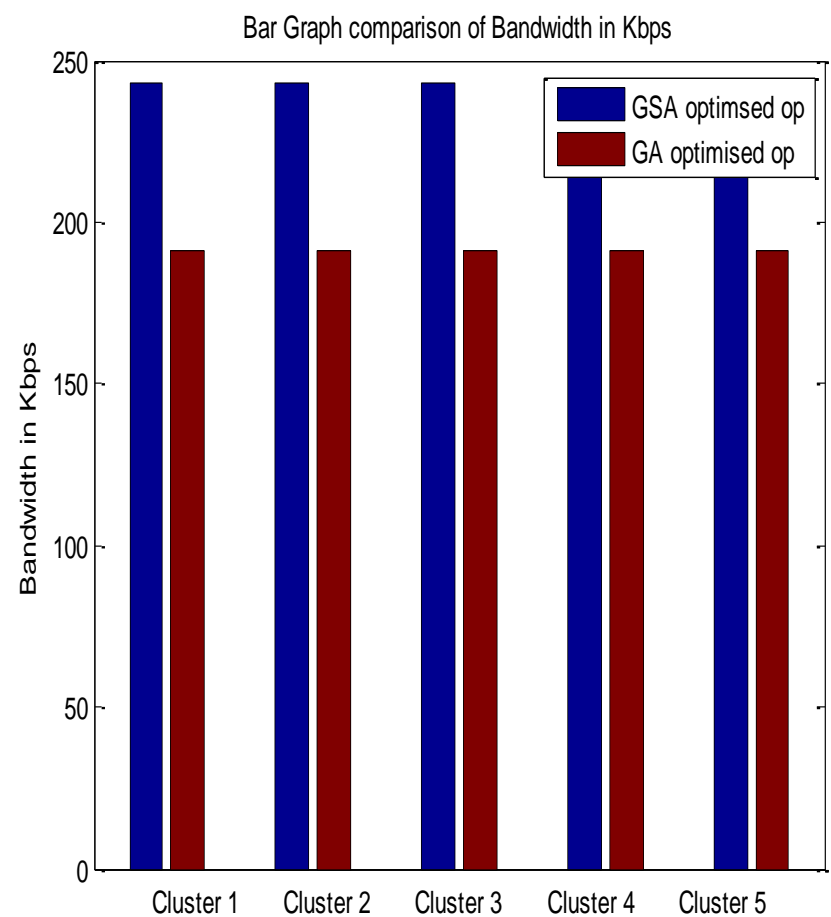

Fig. 3: Bandwidth of cluster heads by proposed and GA optimization

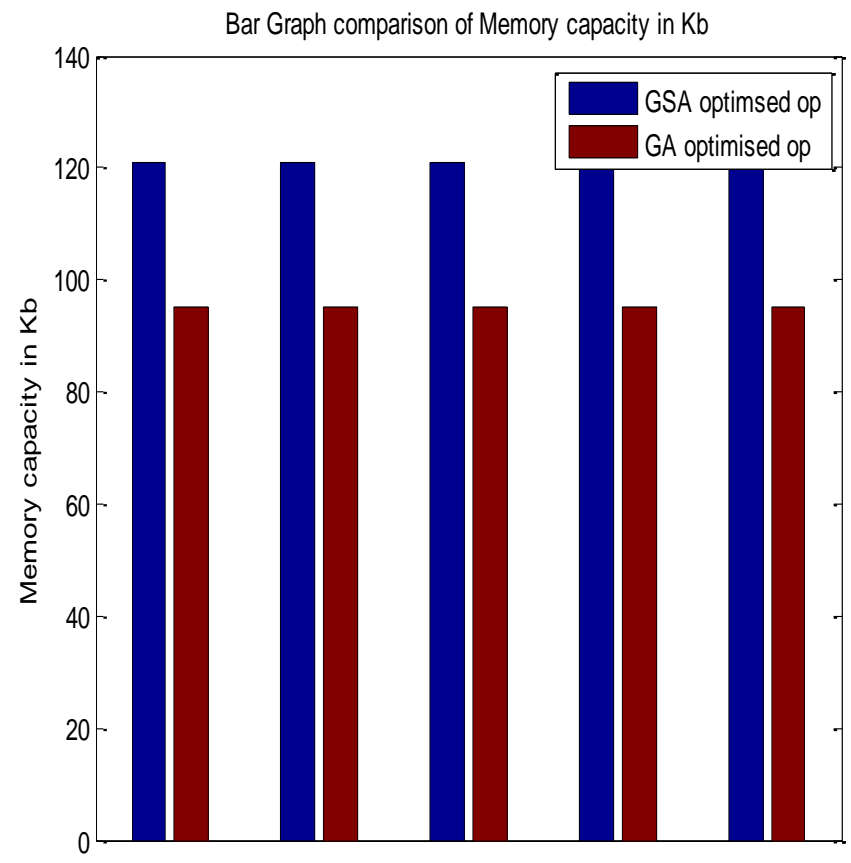

Cluster $1 \quad$ Cluster $2 \quad$ Cluster $3 \quad$ Cluster $4 \quad$ Cluster 5

Fig. 4: Memory left of cluster heads by proposed and GA optimization

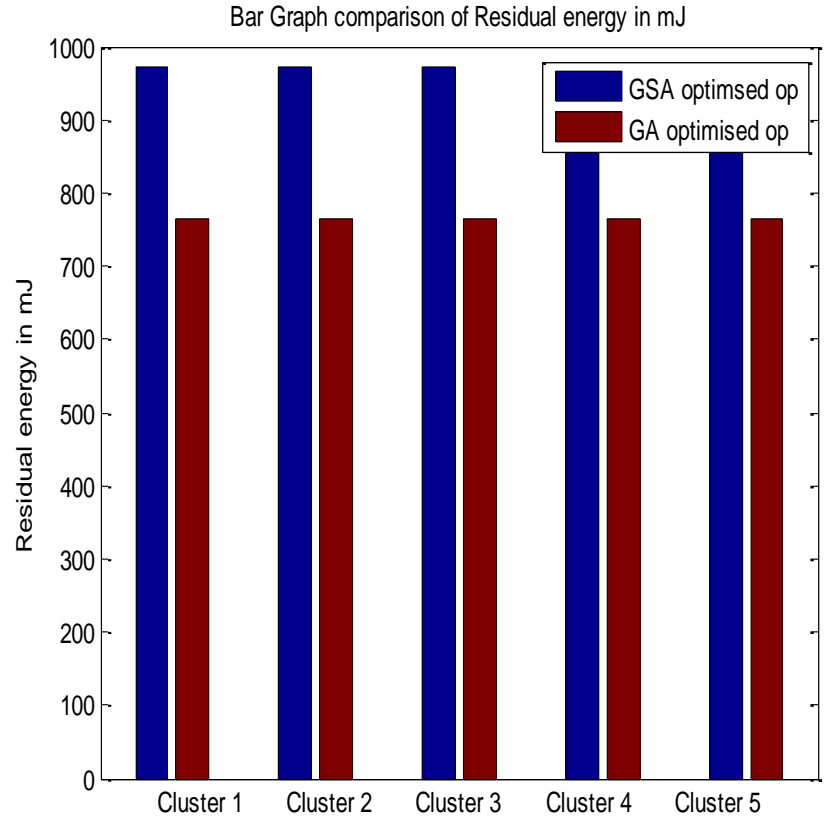

Fig. 5: Residual energy of cluster heads by proposed and GA optimization

It is clear from above graphs that residual energy calculated in cluster heads elected by proposed method is higher than genetic algorithm elected cluster heads[9]. If residual energy is higher that means energy consumption is lower for these. So this method is more energy saver for WSN than previously used GA.

\section{CONCLUSION}

This work is based on reducing the energy consumption in WSN. Here, two tier topology of WSN is considered in which nodes transmit their data to cluster head and which is forwarded to base station by their cluster heads. This way energy consumption is reduced. It can be done either by selecting the cluster head such that energy consumption in making communication with nodes is very less or selecting the optimum path with minimum distance to travel for data packets. In this work both approaches to reduce energy consumption are combined. This is done by using optimization as it is a work of non linear mathematical problems bounded by many constraints. A celestial body inspired optimization algorithm is used which is gravitational search algorithm (GSA). Results are compared with previously used genetic algorithm (GA). Simulation environment of WSN is tested for 100 nodes and three parameters: bandwidth, memory and energy, all are assigned equal to every node. By choosing the cluster head by proposed optimization, remaining values of these three parameters for cluster heads is noted since the node with highest of remaining quantities of these will be elected as cluster head.

\section{FUTURE SCOPE}

A more efficient hybrid algorithm by mixing more than two optimization. A more efficient hybrid algorithm by mixing more than two optimization algorithms can also be tested.This work can be enhanced to provide security to WSN network along with minimizing the energy consumption since security algorithms either run on cluster head or on nodes and consumes energy. 


\section{REFERENCES}

[1] Holger Karl and Andreas Willing (2005), "Protocols and Architectures for Wireless Sensor Network", Text Book, John Wiley \& Sons, Ltd., ISBN: 13 978-0-470-09510-2.

[2] A.S. Uma maheswari, Mrs. S. Pushpalatha," Cluster Head Selection Based On Genetic Algorithm Using AHYMN Approaches in WSN", International Journal of Innovative Research in Science, Engineering and Technology Volume 3, Special Issue 3, March 2014

[3] Fateh boutekkouk, Fatima Taibi, Khawla Meziani "A hybrid approach to extend the life time of heterogeneous wireless sensor networks" The 6th International Conference on Emerging Ubiquitous Systems and Pervasive Networks (EUSPN 2015).

[4] Xuxun Liu, "An optimal-distance based transmission strategy for lifetime maximization of wireless sensor networks", IEEE Sensors journals, Vol.15, No.6, June 2015 .

[5] Emalda Roslin S. and Gomathy C. (2011), "A Novel Topology Control Algorithm for Energy Efficient
Wireless Sensor Network", International Conference on Network and Electronics Engineering (ICNEE 2011), ISBN: 978-981-08-9915-8, IPCSIT,Singapore, Vol.11, pp.76-81.

[6] Junseok Kim, Sokhyeon Chang and Younggoo Kwon (2008),"ODTPC: On-demand Transmission Power Control for Wireless Sensor Networks", IEEE International Conference on Information Networking, ICOIN2008, Seoul, pp.1-5.

[7] Roslin, S.E., "Genetic algorithm based cluster head optimization using topology control for hazardous environment using WSN," in Innovations in Information, Embedded and Communication Systems (ICIIECS), 2015 International Conference on, vol., no., pp.1-7, 19-20 March 2015

[8] E. Rashedi, H. Nezamabadi-pour, and S. Saryazdi, "GSA: A Gravitational Search Algorithm," Information Sciences, vol. 179, no. 13, (2009), pp. 2232-2248.

[9] Jin Fan and Parish D.J. (2007), "Using a Genetic Algorithm to Optimize the Performance of a Wireless Sensor Network", ISBN: 1-9025-6016-7. 